\title{
Intensive versus non-intensive statin pretreatment before percutaneous coronary intervention in Chinese patients: a meta-analysis of randomized controlled trials
}

\section{Xian Yang}

Nanjing University Medical School Affiliated Nanjing Drum Tower Hospital

Xi Lan

Nanjing University Medical School Affiliated Nanjing Drum Tower Hospital

Xin-Lin Zhang

Nanjing University Medical School Affiliated Nanjing Drum Tower Hospital

Zhong-Lin Han

Nanjing University Medical School Affiliated Nanjing Drum Tower Hospital

Si-Min Yan

Nanjing University Medical School Affiliated Nanjing Drum Tower Hospital

Wen-Xiao Wang

Affiliated Hospital of Medical College Qingdao University

Biao Xu

Nanjing University Medical School Affiliated Nanjing Drum Tower Hospital

Wei-Hong Ge ( $\square$ WH.Ge.drumtower@outlook.com )

Nanjing University Medical School Affiliated Nanjing Drum Tower Hospital https://orcid.org/0000-00026124-8742

Research

Keywords: Intensive, Non-Intensive, Statin, Percutaneous Coronary Intervention, Meta-analysis

Posted Date: June 4th, 2020

DOI: https://doi.org/10.21203/rs.3.rs-16709/v2

License: (c) (i) This work is licensed under a Creative Commons Attribution 4.0 International License. Read Full License 


\section{Abstract}

Background: The results of intensive statin pretreatment before percutaneous coronary intervention (PCl) is inconsistent between Chinese and Western populations and there are no corresponding meta-analyses involving hard clinical end-points in the available published literature. The aim of this study was to evaluate the efficacy and safety of high-dose statin loading before $\mathrm{PCl}$ in Chinese patients through a meta-analysis.

Method: Relevant studies were identified by searching the electronic databases of PubMed, Embase, and Cochrane's Library to December 2019. The outcomes included an assessment of major adverse cardiovascular event (MACE), non-fatal myocardial infarction (MI), cardiac death, target vessel revascularization (TVR), myalgia/myasthenia and abnormal alanine aminotransferase (ALT) in all enrolled patients.

Results: 12 studies involving 3,183 individuals were included $\triangle$ The results showed statistically significant different in the incidence of MACE ( $R R=0.49,95 \% \mathrm{Cl}: 0.30-0.80, P=0.004,12=63 \%)$ and non-fatal MI ( $R R=0.54$, $95 \% \mathrm{Cl}: 0.33-0.88, \mathrm{P}=0.01,12=62 \%$ ) on comparing the intensive statin and non-intensive statin treatment groups. Subgroup analysis further suggested the benefits of different treatments were inconsistent. Compared with preoperative intensive statin therapy, the incidence of MACE and non-fatal MI were significantly elevated in patients receiving placebo or no statin treatment before surgery $(\mathrm{RR}=0.47,95 \% \mathrm{Cl}$ : 0.34-0.65, $\mathrm{P}<0.00001, \mathrm{I} 2=0 \%$; RR=0.49, 95\% Cl: 0.35-0.70, $\mathrm{P}<0.0001, \mathrm{I} 2=0 \%$ ). However, the incidence of MACE and non-fatal $\mathrm{Ml}$ were not statistically significant when comparing preoperative high-intensity statin therapy with moderate-intensity statin therapy ( $R R=0.96,95 \% \mathrm{Cl}$ : $0.44-2.08, P=0.91, \mathrm{I}=11 \%$; $R R=1.10,95 \% \mathrm{Cl}$ : 0.86 $1.39, \mathrm{P}=0.44)$. The study also demonstrated that the Asian population could tolerate high-intensity atorvastatin during the perioperative period.

Conclusion: Compared with placebo or no statin pretreatment, Chinese patients receiving intensive statin therapy before PCl displayed reduced incidence of MACE and non-fatal MI. However, there was no significant benefit between high-intensity and moderate-intensity statin treatment. Further, the Chinese population tolerated well preoperative intensive statin pretretment.

\section{Introduction}

As the cornerstone of primary and secondary prevention of arteriosclerotic cardiovascular disease (ASCVD), statin have been widely used in clinical practice. In recent years, several studies have suggested that intensive statin before percutaneous coronary intervention $(\mathrm{PCl})$ can significantly reduce the level of postoperative myocardial damage markers, the incidence of perioperative myocardial infarction and shortterm cardiovascular events ${ }^{[1-3]}$. Current clinical practice guidelines recommend high-dose statin loading before $\mathrm{PCl}$ in Europe and the United States ${ }^{[4-5]}$. However, the research on this field was very limited in Chinese population and no corresponding meta-analysis involving hard clinical endpoints has been published so far.

In addition, there were significant differences in statin metabolism between Chinese and Western people ${ }^{[6]}$. It was unknown whether this would affect the outcome of intensive statin treatment. This paper intended to 
evaluate the efficacy and safety of intensive statin compared with non-intensive statin pretreatment before $\mathrm{PCl}$ in Chinese population through meta-analysis.

\section{Methods}

\section{Search strategy}

A comprehensive search of electronic databases including PubMed, EMBASE, and Cochrane Library was performed. The search was limited from the inception up to December 2019, and of English language. Search terms included "intensive", "intensity", "high", "load", "loading”, "statin", "atorvastatin", "rosuvastatin", "percutaneous coronary intervention", "PCl" and connected using the logical word "AND" or "OR". It was worth mentioning that in order to avoid missing important literatures, retrieval type was not included some terms such as "China" or "Chinese". We checked the location of the research center and the specific inclusion criteria in the article to comprehensively determine the patient was Chinese. The references of the identifed articles and relevant reviews were screened to include other potentially suitable trials.

\section{Inclusion and exclusion criteria}

Studies satisfying the following criteria were eligible: (1) randomized controlled trials (RCTs); (2) the patient was Chinese; (3) the patient with emergency or elective PCl; (4) preoperative interventions for intensive and non-intensive statin therapy which included moderate-intensity statin, placebo and no statin pretreatment; (5) high-intensity statin therapy referred to atorvastatin $\geq 40 \mathrm{mg} / \mathrm{d}$ or rosuvastatin $\geq 20 \mathrm{mg} / \mathrm{d}$ and moderateintensity statin therapy referred to atorvastatin $₫ 40 \mathrm{mg} / \mathrm{d}$ or rosuvastatin $₫ 20 \mathrm{mg} / \mathrm{d}$ or equivalent dose statin; (6) outcome indicators included effectiveness and safety. The former referred to major adverse cardiovascular event (MACE) and the latter referred to myalgia/myasthenia and abnormal alanine aminotransferase (ALT). MACE is defined as cardiac death, nonfatal myocardial infarction and target vessel revascularization. Abnormal ALT is defined as ALT levels that rised more than 3 times the upper limit of normal; (7) follow-up lasted for 1 3 months after PCl; (8) literature language was English. Exclusion criteria included any of the following: chronic high-intensity statin therapy before $\mathrm{PCl}$, abnormal liver enzymes (ALT or aspartate aminotransferase [AST] more than $40 \mathrm{U} / \mathrm{L}$ ); blood creatinine $>2 \mathrm{mg} / \mathrm{dL}$, or history of muscle disease. The studies were reviewed by two independent investigators to determine whether they met the inclusion criteria and any disagreement was resolved by consensus.

\section{Data extraction}

The baseline data involving study characteristics(first author, year of publication, sample size, intervention, follow-up time), patient characteristics(clinical presentation, statin medication history) and outcome indicators were extracted directly from the articles. Differences in assessments were resolved by discussing with a third investigator. 


\section{Quality assessment}

The RCTs were evaluated according to the following methodological criteria recommended by the Cochrane Collaboration: sequence generation, concealment of allocation, blinding, incomplete outcome data, selective outcome reporting, and other sources of bias.

\section{Statistical analysis}

We used the RevMan (Version 5.3; Cochrane Collaboration, Oxford, UK) and Stata software (version 12.0; Stata Corporation, College Station, TX, USA) for the meta-analysis and statistical analysis. Dichotomous data were presented as risk ratios (RR) with $95 \%$ confdence intervals $(\mathrm{Cl})$. The heterogeneity was evaluated using $\mathrm{I}^{2}$ and $p$ value based on Chi-square test. $\mathrm{I}^{2} \leq 50 \%$ or $p \geq 0.1$ did not demonstrate a signifcant heterogeneity and a fixed-effects model was used. $1^{2} \rrbracket 50 \%$ or $p \bowtie 0.1$ indicated a signifcant heterogeneity, and therefore, a random-effects model was applied. Subgroup analysis was carried out to explore the sources of heterogeneity. Sensitivity analyses were performed by excluding sequentially one study at a time to test the robustness of the results. Potential publication bias was assessed with a funnel plot and Egger's regression asymmetry test. All $p$ values were two-sided, and results were considered statistically signifcant when the

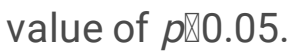

\section{Results}

\section{Study selection and quality assessment}

As shown in Fig 1, 4020 potentially relevant articles were identifed in the initial analysis. Among them, a total of 3391 articles were identified after removal of duplicate studies. Only 27 articles were retained after screening the title and abstract. Finally, 12 studies involving 3183 patients were included in the present meta-analysis ${ }^{[7-18]}$. Among them, 1545 patients belonged to intensive statin treatment group and 1638 patients belonged to non-intensive statin treatment group. Further more, non-intensive statin treatment group involved moderateintensity statin, placebo and no statin pretreatment group which included 738, 244 and 656 patients, respectively. All patients were female in one study ${ }^{[16]}$. The characteristics of the included studies were shown in Table 1. The baseline clinical, angiographic and procedural characteristics of patients are listed in Table 2. Quality assessment results were described in Table 3. 


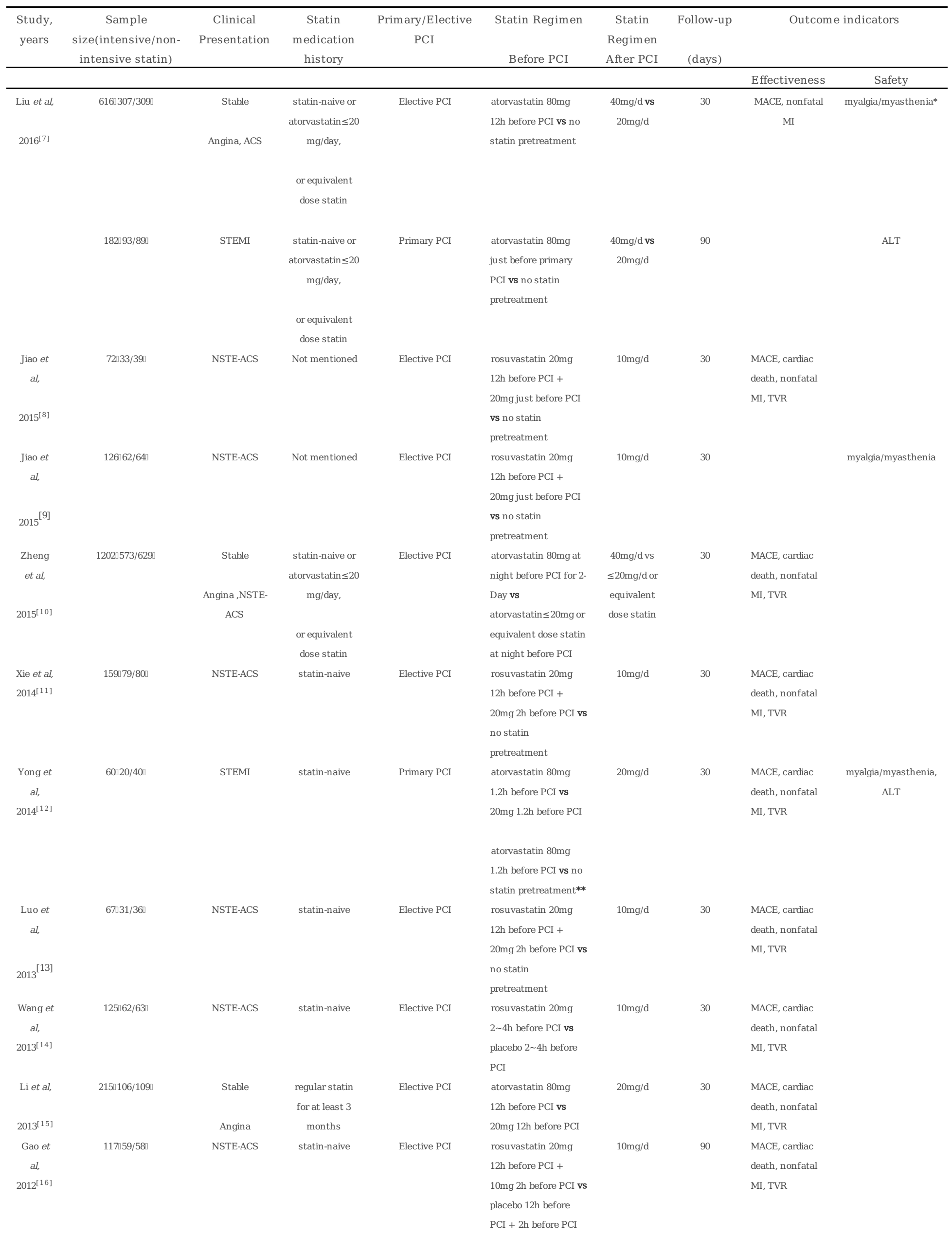

Page 5/18 


\begin{tabular}{|c|c|c|c|c|c|c|c|c|c|}
\hline \multirow[t]{2}{*}{ Li et al, } & $161 \unlhd 78 / 83 \square$ & STEMI & statin-naive & Primary PCI & atorvastatin $80 \mathrm{mg}$ & $40 \mathrm{mg} / \mathrm{d}$ & 30 & & ALT \\
\hline & & & & & $1.5 \mathrm{~h}$ before $\mathrm{PCI}$ vs & & & & \\
\hline \multirow[t]{2}{*}{$2012^{[17]}$} & & & & & placebo $1.5 \mathrm{~h}$ before & & & & \\
\hline & & & & & PCI & & & & \\
\hline \multirow[t]{2}{*}{ Yu et al, } & $81 \square 41 / 40 \square$ & NSTE-ACS & statin-naive & Elective PCI & atorvastatin $80 \mathrm{mg}$ & $20 \mathrm{mg} / \mathrm{d}$ & 30 & MACE, cardiac & \\
\hline & & & & & 12h before PCI + & & & death, nonfatal & \\
\hline \multirow[t]{3}{*}{$2011^{[18]}$} & & & & & $40 \mathrm{mg} 2 \mathrm{~h}$ before $\mathrm{PCI}$ vs & & & MI, TVR & \\
\hline & & & & & placebo $12 \mathrm{~h}$ before & & & & \\
\hline & & & & & PCI $+2 \mathrm{~h}$ before $\mathrm{PCI}$ & & & & \\
\hline
\end{tabular}

year; **Moderate-intensity group and no statin pretreatment group included 20 patients separately

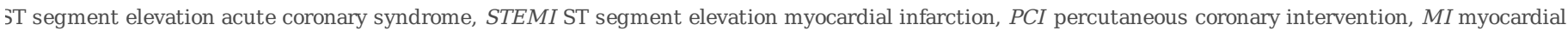
:arget vessel revascularization

e 2 Baseline clinical, angiographic and procedural characteristics in the overall population

\begin{tabular}{|c|c|c|c|}
\hline riable & $\begin{array}{l}\text { High-intensity statin } \\
\text { n/population (\%) }\end{array}$ & $\begin{array}{c}\text { Moderate-intensity statin } \\
\text { n/population (\%) }\end{array}$ & $\begin{array}{l}\text { Placebo or no statin pretreatment } \\
\text { n/population (\%) }\end{array}$ \\
\hline mber of patients & $1544 / 3183(48.5 \%)$ & $758 / 3183(23.8 \%)$ & $881 / 3183(27.7 \%)$ \\
\hline$\underline{\text { le }}$ & $1070 / 1544(69.3 \%)$ & $536 / 758(70.7 \%)$ & $582 / 881(66.1 \%)$ \\
\hline pertension & $956 / 1544(61.9 \%)$ & $501 / 758(66.1 \%)$ & $544 / 881(61.7 \%)$ \\
\hline betes Mellitus & $443 / 1449(30.6 \%)$ & $234 / 758(30.9 \%)$ & $251 / 778(32.3 \%)$ \\
\hline slipidaemia & $201 / 771(26.1 \%)$ & $152 / 649(23.4 \%)$ & 78/201(38.8\%) \\
\hline okers & $552 / 1544(35.8 \%)$ & $293 / 758(38.7 \%)$ & $297 / 881(33.7 \%)$ \\
\hline vious MI & 99/630(15.7\%) & $9 / 20(45.0 \%)$ & 96/632(\%) \\
\hline vious PCI & $159 / 1183(\%)$ & $51 / 629(\%)$ & $103 / 612(15.2 \%)$ \\
\hline vious CABG & $6 / 500(1.2 \%)$ & $0 / 0(0 \%)$ & $6 / 496(1.2 \%)$ \\
\hline ble angina & $230 / 506(45.5 \%)$ & $109 / 109(100 \%)$ & $118 / 398(29.6 \%)$ \\
\hline TE-ACS & $518 / 767(67.5 \%)$ & $0 / 0(0 \%)$ & $539 / 778(69.3 \%)$ \\
\hline $\mathrm{MI}$ & $223 / 498(44.8 \%)$ & $20 / 20(100 \%)$ & $224 / 501(44.7 \%)$ \\
\hline gle vessel & $84 / 266(31.6 \%)$ & $6 / 20(30.0 \%)$ & $80 / 279(28.7 \%)$ \\
\hline uble vessel & $106 / 266(39.8 \%)$ & $4 / 20(20.0 \%)$ & $111 / 279(39.8 \%)$ \\
\hline re than three and triple vessel & $81 / 266(30.5 \%)$ & $10 / 20(50.0 \%)$ & $95 / 279(34.1 \%)$ \\
\hline getvessel LM & $36 / 877(4.1 \%)$ & $25 / 629(4.0 \%)$ & $7 / 320(2.2 \%)$ \\
\hline get vessel LAD & $634 / 1038(61.1 \%)$ & $427 / 649(65.8 \%)$ & $261 / 483(54.0 \%)$ \\
\hline getvessel LCX & $330 / 1038(31.8 \%)$ & $201 / 649(31.0 \%)$ & $159 / 483(32.9 \%)$ \\
\hline get vessel RCA & $352 / 1038(33.9 \%)$ & $243 / 649(37.4 \%)$ & $168 / 483(34.8 \%)$ \\
\hline 'C lesions & $346 / 609(56.8 \%)$ & $0 / 0(0 \%)$ & $333 / 615(54.1 \%)$ \\
\hline ltivessel lesions & $33 / 78(42.3 \%)$ & $0 / 0(0 \%)$ & $39 / 83(47.0 \%)$ \\
\hline ltivessel intervention & $244 / 773(31.6 \%)$ & $215 / 629(34.2 \%)$ & $62 / 201(30.8 \%)$ \\
\hline jirin & $1375 / 1491(92.2 \%)$ & $622 / 738(84.3 \%)$ & $802 / 822(97.6 \%)$ \\
\hline pidogrel/Ticlopidine & $1300 / 1385(93.9 \%)$ & $541 / 629(86.0 \%)$ & $807 / 822(98.2 \%)$ \\
\hline lockers & $1104 / 1491(74.0 \%)$ & $495 / 738(67.1 \%)$ & $630 / 822(76.6 \%)$ \\
\hline EI/ARB & $1045 / 1491(70.1 \%)$ & $404 / 738(54.7 \%)$ & $667 / 822(81.1 \%)$ \\
\hline coprotein IIb/IIIa inhibitors & $95 / 370(25.7 \%)$ & $9 / 20(45.0 \%)$ & $113 / 380(29.7 \%)$ \\
\hline $\mathrm{S}$ & $822 / 851(96.6 \%)$ & $701 / 738(95.0 \%)$ & $176 / 179(98.3 \%)$ \\
\hline
\end{tabular}

us MI previous myocardial infarction, Previous PCI previous percutaneous coronary intervention, Previous CABG Previous Coronary artery bypass ing, NSTE-ACS non-ST segment elevation acute coronary syndrome, STEMI ST segment elevation myocardial infarction, $L M$ left main, $L A D$ left anterior descending, eft circumflex, $R C A$ right coronary artery, $D E S$ drug-eluting stent 
Table 3 Assessment of randomized controlled trials

\begin{tabular}{|c|c|c|c|c|c|c|}
\hline Study, year & Randomization & Allocation & Blinding of participants, & Incomplete outcome & Selective & Other \\
\hline & sequence generation & concealment & personnel and outcome & data & reporting & sources \\
\hline & & & assessors & & & of bias \\
\hline Liu et al, 2016[7] & Low risk & Low risk & High risk & Unclear risk & Unclear risk & Unclear risk \\
\hline Jiao et al, 2015 ${ }^{[8]}$ & Unclear risk & Unclear risk & Unclear risk & Unclear risk & Unclear risk & Unclear risk \\
\hline Jiao et al, 2015 & Low risk & Low risk & Unclear risk & Unclear risk & Unclear risk & Unclear risk \\
\hline Zheng et al, $2015^{[10]}$ & Unclear risk & Unclear risk & High risk & Low risk & Low risk & Unclear risk \\
\hline Xie et al, 2014 $4^{[11]}$ & Unclear risk & Unclear risk & Unclear risk & Unclear risk & Unclear risk & Unclear risk \\
\hline Yong et al, 2014 ${ }^{[12]}$ & Unclear risk & Unclear risk & Unclear risk & Unclear risk & Unclear risk & Unclear risk \\
\hline Luo et al, 2013 ${ }^{[13]}$ & Unclear risk & Unclear risk & Unclear risk & Unclear risk & Unclear risk & Unclear risk \\
\hline Wang et al, $2013^{[14]}$ & Unclear risk & Unclear risk & Unclear risk & Unclear risk & Unclear risk & Unclear risk \\
\hline Li et al, $2013^{[15]}$ & Low risk & Low risk & Low risk & Unclear risk & Unclear risk & Unclear risk \\
\hline Gao et al, 2012 ${ }^{[16]}$ & Unclear risk & Unclear risk & High risk & Unclear risk & Unclear risk & Unclear risk \\
\hline Li et al, $2012^{[17]}$ & Unclear risk & Unclear risk & Low risk & Unclear risk & Unclear risk & Unclear risk \\
\hline Yu et al, $2011^{[18]}$ & Unclear risk & Unclear risk & Unclear risk & Unclear risk & Unclear risk & Unclear risk \\
\hline
\end{tabular}

\section{Effectiveness analysis}

There were 10 studies that compared the effects of preoperative intensive statin therapy and non-intensive statin therapy on the incidence of MACE and nonfatal MI respectively ${ }^{[7-8,10-}$ $16,18]$. Due to the heterogeneity between different results, a random effects model was used. The results showed the incidence of MACE ( $R R=0.49,95 \% C I: 0.30 \sim 0.80, p=0.004, \mathrm{I}^{2}=63 \%$, Fig. 2a) and nonfatal MI ( $R R=0.54,95 \% C I$ : 0.33 0.88, $p=0.01, \mathrm{I}^{2}=62 \%$, Fig. $2 \mathrm{~b}$ ) between two groups were statistically significant. There were 9 studies that compared the effects of preoperative intensive statin therapy and non-intensive statin therapy on the incidence of cardiac death and target vessel revascularization ${ }^{[8,10-16,18]}$. Considering the homogeneity between the results, a fixed effect model was used and both indicators were not statistically significant $R R=0.37,95 \% C I: 0.01 \sim 8.96, p=0.54$, Fig. 2c; $R R=0.43,95 \% C I: 0.18 \sim 1.02, p=0.06$, $\mathrm{I}^{2}=0 \%$, Fig. $2 \mathrm{~d}$. 
There were 3 studies that compared the effects of preoperative intensive statin therapy and non-intensive statin therapy on the incidence of myalgia/myasthenia ${ }^{[7,9,12]}$ and abnormal $\mathrm{ALT}^{[7,12,17]}$ respectively. No significant difference was observed between two groups $\square R R=1.35,95 \% C I: 0.30 \sim 5.95, p=0.69, \mathrm{I}^{2}=0 \%$, Fig. 3a; $R R=1.47,95 \% C I: 0.54 \sim 4.02, p=0.45$, $\mathrm{I}^{2}=0 \%$, Fig. 3b口.

\section{Subgroup analysis according to intensive statin therapy}

Due to the heterogeneity in MACE and nonfatal MI for the overall results, a subgroup analysis was attempted to find a source of heterogeneity. It was performed by dividing the nonintensive statin treatment group into moderate-intensity ${ }^{[10,12,15]}$ and placebo/no statin treatment group ${ }^{[7,8,11-14,16,18]}$ involving 10 studies. The results showed that the heterogeneity has disappeared in both two subgroup. Compared with the preoperative intensive statin therapy, the incidence of MACE and nonfatal MI were significantly elevated in patients receiving placebo or no statin treatment before surgery $R R=0.47,95 \% C I$ : $0.34 \sim 0.65$, $p<0.00001, \mathrm{I}^{2}=0 \%$, Fig. 2a; $R R=0.49,95 \% C I$ : 0.35 0.70, $p<0.0001, \mathrm{I}^{2}=0 \%$, Fig. 2b口. These findings were consistent with the general population. However, the incidence of MACE and nonfatal MI were not statistically significant compared preoperative high-intensity statin therapy with moderate-intensity statin therapy $R R=0.96,95 \% C I: 0.44 \sim 2.08, p=0.91, \mathrm{I}^{2}=11 \%$, Fig. 2a; $R R=1.10,95 \% C I$ : 0.86 1.39, $p=0.44$, Fig. $2 \mathrm{~b} \square$. The results for this subgroup were inconsistent with overall results and could explain the cause of heterogeneity.

\section{Sensitivity analysis}

Considering the results of the subgroup were inconsistent with overall population, sensitivity analysis conducted further through the removal of any single trial. The heterogeneity of general population in MACE and nonfatal MI no longer existed when excluding the study by Zheng et $a \rrbracket R R=0.46,95 \% C I: 0.33 \sim 0.65, p<0.00001, \mathrm{I}^{2}=0 \%$, Additional file 1: Figure S1a; $R R=0.49,95 \% C I: 0.35 \sim 0.70, p<0.0001, \mathrm{I}^{2}=0 \%$, Additional file 1: Figure S1b־and it did not essentially affect the results of pooled general population and subgroup ${ }^{[10]}$.

\section{Publication bias}

The plots were symmetrical on visual inspection, indicating risk of publication bias (Fig.4). Egger's regression test also demonstrated risk of publication bias ( $p=0.001$, Fig.5). The small number of studies included in the overall population and subgroup may be one of the reasons for publication bias ${ }^{[19]}$.

\section{Discussion}


Some studies have been completed on intensive statin therapy before PCI. In 2013, the ALPACS study took the lead in exploration in Asia ${ }^{[20]}$. It was a prospective, multicenter, randomized, open-label study involving 499 patients with NSTE-ACS(26 clinical centers in China and South Kore). None of the enrolled patients had previously received statin. The intensive treatment group received additional atorvastatin loading doses of $80 \mathrm{mg}$ at $12 \mathrm{~h}$ and $40 \mathrm{mg}$ at $2 \mathrm{~h}$ pre-PCl. The conventional treatment group was only treated with atorvastatin $40 \mathrm{mg} / \mathrm{d}$ after $\mathrm{PCl}$. The results suggested the intensive treatment group failed to significantly reduce the occurrence of MACE at 30 days after $\mathrm{PCl}$ compared with the conventional treatment group (15.7\% vs $14.7 \%, p=0.80)$. The study also demonstrated Asian population were able to tolerate high-intensity atorvastatin during the perioperative period. Unfortunately, the ALPACS were not included in this metaanalysis because of the mixed data from the Korean population. The ISCAP study subsequently published in 2015 was a large-scale, multicenter, randomized, prospective, open-label, blinded, parallel controlled clinical study with Chinese patients ${ }^{[10]}$. Follow-up results showed no significant difference in the incidence of MACE at 30 days between the intensive statin treatment group and the conventional treatment group (19.4\% vs $18.3 \%, P=0.43)$. Followed up for 6 months, there was still no difference between the two groups (20.1\% vs $18.3 \%, P=0.63)$. In terms of safety, no significant differences were found in liver enzymes, creatine kinase and other indicators.

In addition to multi-center clinical studies, many scholars tried to find further answers with meta-analysis. In 2013, Guo et al.conducted a meta-analysis on the impact of sequential statin therapy on the prognosis of Chinese patients with PCI ${ }^{[21]}$. 10 studies involving 1015 patients were included in this article. The results suggested a significant reduction in the incidence of MACE within 6 months. Since some patients in experimental group just received intensive statin treatment after $\mathrm{PCl}$, the subjects were not entirely consistent with the characteristics discussed in this paper. In 2017, a systematic review and meta-analysis involving 11 RCTs with 802 patients was performed by Ye et al[22] Compared with preoperative rosuvastatin 10mg/d, using loading dose of $20 \mathrm{mg} / \mathrm{d}$ before $\mathrm{PCl}$ could significantly reduce cTnT and hs-CRP levels 24 hours and LDL-C, TC, and TG levels 30 days after PCl. However, the clinical indicators analyzed and evaluated in this article were surrogate indicators, which did not involve cardiovascular end point events, nor did they examine the safety. In 2018, Cao et al. discussed the effectiveness of high-dose statin before PCl in reducing cardiovascular events in Asian populations ${ }^{[23]}$. The systematic review included 7 RCTs involving 1381 patients, all of whom were Chinese or Korean. The analysis results indicated that the incidence of MACE and perioperative myocardial infarction in the intensive statin group were significantly lower than those in the control group. This article did not discuss the benefits of the Chinese population through subgroup analysis.

Compared with the previously published meta-analysis, this article was improved in the following aspects. First of all, four new studies published after 2014 were included in this paper ${ }^{[7-10]}$. The full paper included 12 RCTs with 3183 patients. It meant the total number of studies as well as patients exceeded any previously published meta-analysis. Secondly, the population involved in this paper was all Chinese, so the interference of other Asian populations such as Korean population was removed. Thirdly, new outcome indicators such as cardiac death and target vessel revascularization were established to make the data more complete.

An important finding of this study was that the subgroup analysis could eliminate the heterogeneity of the overall population, which suggested the benefits of different treatment were not consistent. On the one hand, 
people received high-intensity statin could benefit from patients received placebo or no statin treatment before $\mathrm{PCl}$. This conclusion was consistent with the results of previous studies involving Western populations. On the other hand, there were no statistically differences in MACE and other outcome indicators between patients received high-intensity and patients received moderate-intensity statin preoperatively, which suggested two regimens had a consistent effect on short-term outcomes. We further searched the database and found the lack of RCTs of two regimens in Western populations at present. In fact, there have been reports of racial differences in the pharmacokinetics of statin. With a single dose of $20 \mathrm{mg}$ or $40 \mathrm{mg}$ rosuvastatin, the area under the curve and peak blood concentration of Chinese were 1.79, 1.89, 2.31, and 2.36 times that of Caucasians, respectively ${ }^{[24-25]}$. Differences in race sensitivity to statin may be related to genetic factors. Genetic differences in metabolic enzymes and drug transporters between Chinese and white may partially explain this phenomenon ${ }^{[6]}$. However, based on the results of this paper and previous studies, we did not find any racial differences on the efficacy and safety of preoperative intensive statin therapy.

The sensitivity analysis of this study suggested that the results of the ISCAP was the source of heterogeneity which was the largest clinical study to date targeting Chinese patients. Although negative results were obtained, we also noticed there might be some confounding factors that affected the final conclusions. Firstly, about $60 \%$ patients enrolled had previously taken low-intensity even moderate-intensity statin and only $40 \%$ were statin-naive patients. It was unclear whether statin history could reduce the benefits from intensive treatment in Chinese patients. Secondly, the time of drug administration in the ISCAP was at night before the operation and it was not exactly fixed. Different from it, the time were 2 4 hours or 12 hours before elective $\mathrm{PCl}$ and relatively fixed in other trials ${ }^{[7-9,11,13-16,18]}$. On the one hand, the regimen in the ISCAP was more consistent with clinical practice. On the other hand, it was uncertain whether the time of administration could affect the benefits of intensive statin. Finally, characteristics of patients enrolled in the ISCAP with higher proportion of multiple lesions, higher average number of stents, longer average length of stents and higher incidence of MACE at 30 days after PCl indicated that the complexity of coronary lesion might reduce the effectiveness of high-dose statin treatment.

The guidelines and consensuses underwent a process of deepening understanding on whether the patients should receive preoperative intensive statin therapy in Chinese. Based on evidence for Western populations, expert consensus released in 2014 recommended all patients with acute coronary syndrome (ACS) undergoing $\mathrm{PCl}$, including emergency and elective $\mathrm{PCl}$, should initiate high-dose statin treatment immediately before $\mathrm{PCl}$, such as atorvastatin $80 \mathrm{mg} / \mathrm{d}^{[26]}$. As ALPACS and ISCAP published in succession, guidelines issued in 2016 concluded that in the absence of more evidence of high-quality RCTs with hard endpoints, it was not recommended patients with ACS received intensive statin therapy before $\mathrm{PCl}^{[27-28]}$. The results of this meta-analysis were consistent with the recommendations of the guidelines and further strengthen the foundation of evidence-based medicine.

\section{Study limitations}


This article has the following limitations and deficiencies: (1) The quality of included studies were generally not high. The remaining 11 RCTs were single-center and lacked rigorous trial design except ISCAP. The randomization, blinding and data analysis of these trials were not described in detail. We also need to noticed that ISCAP was an open-label trial. Although it had all outcome events adjudicated by a blind CEC group and all laboratory tests have been done by blind laboratory staff, the investigators and subjects were not blinded to the treatment arms of this trial, and as such, both might tend to report more adverse events in the experiment arm and this might lead to more withdrawal from study in this group. This trend could still have some affects on the results of the study. (2) Subgroup analysis was not comprehensive. Limited by the included literatures, this meta-analysis only performed a subgroup analysis based on the intensity of statin without involving other factors, such as statin medication history (patients with chronic statin therapy or statin-naive patients), categories of statin (atorvastatin or rosuvastatin), timing of statin administration (12 hours before surgery or other time), timing of revascularization (emergency $\mathrm{PCl}$ or elective $\mathrm{PCl}$ ). (3) Due to the lack of RCTs received moderate and high-intensity statin before PCl in Western populations, the effects of race differences on statin pretreatment could not be further analyzed.

\section{Conclusions}

Available evidence suggested, compared with placebo or no statin pretreatment, Chinese patients received intensive statin before $\mathrm{PCl}$ can further reduce the incidence of MACE and nonfatal MI. However there was no significant benefit between high-intensity and moderate-intensity statin. In addition, the Chinese population was well tolerated by preoperative intensive statin pretretment.

\section{Additional File}

Figure S1. Forest plot of MACE and nonfatal MI with preoperative intensive statin therapy and non-intensive statin therapy in patients when excluding the study by Zheng et al.

\section{Abbreviations}

PCI: Percutaneous coronary intervention; MACE: Major adverse cardiac events; MI: Myocardial infarction; TVR: Target vessel revascularization; ALT: Alanine aminotransferase; ASCVD: Arteriosclerotic cardiovascular disease; RCTs: Randomizedcontrolled trials; AST: Aspartate aminotransferase; RR: Risk ratios; Cl: Confidence intervals; NSTE-ACS: Non-ST segment elevation acute coronary syndrome; STEMI: ST segment elevation myocardial infarction; CABG: Coronary artery bypass grafting; LM: Left main; LAD: Left anterior descending; LCX: Left circumflex; RCA: Right coronary artery; DES: Drug-eluting stent; ACS: Acute coronary syndrome.

\section{Declarations}

\section{Acknowledgements}

Not applicable. 


\section{Authors' contributions}

WHG and XY conceived of the study, participated in the design, and drafted the manuscript. XL and XLZ carried out the study searches, XL, ZLH and SMY collected the data. WXW and BX performed the statistical analyses. All authors reviewed and revised the manuscript, and approved the manuscript for submission.

\section{Funding}

No funding was received for this study.

\section{Availability of data and materials}

All data generated or analysed during this study are included in this published article.

\section{Ethics approval and consent to participate}

Not applicable.

\section{Consent for publication}

Not applicable.

\section{Competing interests}

The authors declare that they have no competing interests.

\section{Author details}

${ }^{1}$ Department of Pharmacy, Nanjing Drum Tower Hospital, The Affiliated Hospital of Nanjing University Medical School, No. 321 Zhongshan Road, Nanjin 210008, China. ${ }^{2}$ Department of Cardiology, Nanjing Drum Tower Hospital, The Affiliated Hospital of Nanjing University Medical School, Nanjin 210008, China.

${ }^{3}$ Department of Pharmacy, The Affiliated Hospital of Qingdao University, Qingdao 266000, China.

\section{References}


1. Di SG 1, Patti G, Pasceri V, Gaspardone A, Colonna G, Montinaro A. Efficacy of atorvastatin reload in patients on chronic statin therapy undergoing percutaneous coronary intervention: results of the ARMYDA-RECAPTURE (Atorvastatin for Reduction of Myocardial Damage During Angioplasty) Randomized Trial. J Am Coll Cardiol. 2009;54:558-65.

2. Patti G, Cannon CP, Murphy SA, Mega S, Pasceri V, Briguori C, et al. Clinical benefit of statin pretreatment in patients undergoing percutaneous coronary intervention: a collaborative patient-level meta-analysis of 13 randomized studies. Circulation. 2011;123:1622-32.

3. Briguori C, Visconti G, Focaccio A, Golia B, Chieffo A, Castelli A, et al. Novel approaches for preventing or limiting events (Naples) II trial: impact of a single high loading dose of atorvastatin on periprocedural myocardial infarction. J Am Coll Cardiol. 2009;54:2157-63.

4. Catapano AL, Graham I, De BG, Wiklund O, Chapman MJ, Drexel H, et al. 2016 ESC/EAS Guidelines for the management of dyslipidaemias:The task force for the management of dyslipidaemias of the European society of cardiology (ESC) and European atherosclerosis society (EAS) 『Eur Heart J. 2016;37:2999-3058.

5. 5. Levine GN, Bates ER, Blankenship JC, Bailey SR, Bittl JA, Cercek B, et al. 2011 ACCF/AHA/SCAI guideline for percutaneous coronary intervention. A report of the American College of Cardiology Foundation/American Heart Association Task Force on Practice Guidelines and the Society for Cardiovascular Angiography and Interventions. J Am Coll Cardiol. 2011;58:e44-e122.

6. Naito R, Miyauchi K, Daida H. Racial Differences in the Cholesterol-Lowering Effect of Statin. J Atheroscler Thromb. 2017;24:19-25.

7. Liu Z, Herrmann J, Hao HJ, Xu J, Hu SD, Li BY, et al. Efficacy of High-Intensity Atorvastatin for Asian Patients Undergoing Percutaneous Coronary Intervention. Ann Pharmacother. 2016;50:725-33.

8. Jiao YG, Hu F, Zhang ZG, Gong KZ, Sun XN, Li AH, et al. Effect of rosuvastatin dose-loading on serum sLox-1, hs-CRP, and postoperative prognosis in diabetic patients with acute coronary syndromes undergoing selected percutaneous coronary intervention (PCI) . Int J Clin Exp Med. 2015;8,21565-71.

9. Jiao YG, Hu F, Zhang ZG, Gong KZ, Sun XN, Li AH, et al. Efficacy and Safety of Loading-Dose Rosuvastatin Therapy in Elderly Patients with Acute Coronary Syndromes Undergoing Elective Percutaneous Coronary Intervention . Clin Drug Investig. 2015;35:777-84.

10. Zheng B, Jiang J, Liu HL, Zhang J, Li H, Su L, Wang HC, et al. Efficacy and safety of serial atorvastatin load in Chinese patients undergoing elective percutaneous coronary intervention: results of the ISCAP (Intensive Statin Therapy for Chinese Patients with Coronary Artery Disease Undergoing Percutaneous Coronary Intervention) randomized controlled trial. Eur Heart J Suppl. 2015;17 (Supplement B) :B47-B56.

11. Xie WC, Li P, Wang ZD, Chen J, Lin ZH, Liang XW, et al. Rosuvastatin may reduce the incidence of cardiovascular events in patients with acute coronary syndromes receiving percutaneous coronary intervention by suppressing miR-155/SHIP-1 signaling pathway. Cardiovasc Ther. 2014;32:276-82.

12. Yong HJ, Wang X, Mi L, Guo LJ, Gao W, Zhang YZ, et al. Effects of atorvastatin loading prior to primary percutaneous coronary intervention on endothelial function and inflammatory factors in patients with ST-segment elevation myocardial infarction. Exp Ther Med. 2014;7:316-22. 
13. Luo J, Li J, Shen XQ, Hu XQ, Fang ZF, Lv XL, et al. The effects and mechanisms of high loading dose rosuvastatin therapy before percutaneous coronary intervention in patients with acute coronary syndrome . Int J Cardiol. 2013;167:2350-3.

14. Wang ZZ, Dai HY, Xing MQ, Yu ZX, Lin XR, Wang SD, et al. Effect of a single high loading dose of rosuvastatin on percutaneous coronary intervention for acute coronary syndromes. J Cardiovasc Pharmacol Ther. 2013;18:327-33.

15. Li Q, Deng SB, Xia S, Du JL, She Q. Impact of intensive statin use on the level of inflammation and platelet activation in stable angina after percutaneous coronary intervention: a clinical study. Med Clin (Barc). 2013;140:532-6.

16. Gao Y, Jia ZM, Sun YJ, Zhang ZH, Ren LN, Qi GX, et al. Effect of high-dose rosuvastatin loading before percutaneous coronary intervention in female patients with non-ST-segment elevation acute coronary syndrome. Chin Med J (Engl). 2012;125:2250-4.

17. Li W, Fu XH, Wang YB, Li XN, Yang ZX, Wang XC, et al. Beneficial effects of high-dose atorvastatin pretreatment on renal function in patients with acute ST-segment elevation myocardial infarction undergoing emergency percutaneous coronary intervention. Cardiology. 2012;122:195-202.

18. Yu XL, Zhang HJ, Ren SD, Geng J, Wu TT, Chen XQ, et al. Effects of loading dose of atorvastatin before percutaneous coronary intervention on periprocedural myocardial injury. Coron Artery Dis. 2011;22:8791.

19. Jang YS, Zhu JR, Ge JB, Kim YJ, Ji C, Lam W. Preloading with atorvastatin before percutaneous coronary intervention in statin-naïve Asian patients with non-ST elevation acute coronary syndromes: A randomized study. Coron Artery Dis. 2011;22:87-91.

20. Guo XY. A meta-analysis of sequential therapy of statins on PCl patients in china. CNKI. 2019. https://www.cnki.net/.

21. Ye ZL, Lu HL, Su Q, Guo WQ, Dai WR, Li HQ, et al. Effect of high-dose rosuvastatin loading before percutaneous coronary intervention in Chinese patients with acute coronary syndrome: A systematic review and meta-analysis. Plos One. 2017,12:e0171682.

22. Cao AL, Qian J, Wang Z. Efficacy of high-dose statin administration before surgery on the reduction of cardiovascular events: a meta analysis. Pharm Care Res. 2018;18:282-7.

23. 2 Birmingham BK, Bujac SR, Elsby R, Azumaya CT, Zalikowski J, Chen YS, et al. Rosuvastatin pharmacokinetics and pharmacogenetics in Caucasian and Asian subjects residing in the United States. Eur J Clin Pharmacol. 2015;71:329-40.

24. Lee E, Ryan S, Birmingham B, Zalikowski J, March R, Ambrose H, et al. Rosuvastatin pharmacokinetics and pharmacogenetics in white and Asian subjects residing in the same environment. Clin Pharmacol Ther, 2005;78:330-41.

25. Huo Y, Ge JB, Han YL, Wang JA, Wan Z, Li JP, et al. Expert consensus on intensive statin therapy for patients with acute coronary syndrome. Chin J Intervent Cardiol. 2014;22:4-6.

26. Joint committee issued Chinese guideline for the management of dyslipidemia in adults. Chinese guideline for the management of dyslipidemia in adults. Chin Circ J. 2016;32:937-53. 
27. Chinese Medical Association in cardiovascular disease branch interventional cardiology group, Chinese Medical Doctor Association cardiovascular physician branch thrombus prevention and control Specialized Committee, Chinese Journal of cardiovascular disease editorial board. Guidelines for percutaneous coronary intervention in China (2016). Chin J Cardiol. 2016;44:382-400.

\section{Figures}

4020 Citations retrieved from database search

602 Duplicate records excluded

Articles after duplicates removed for review

3391 Articles excluded based on title, abstract or both

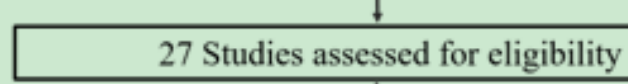

27 Studies assessed for eligibility

15 Studies excluded based on:

1 endpoints included cerebrovascular events

1 enrolled patients did not receive PCI

1 enrolled patients did not receive intensive statin before PCI

2 control group also received the loading dose

2 enrolled patients included Koreans

8 insufficient data

12 RCTs included in meta-analysis

Figure 1

Flowchart of literature search for this meta-analysis. PCl: Percutaneous coronary intervention. 

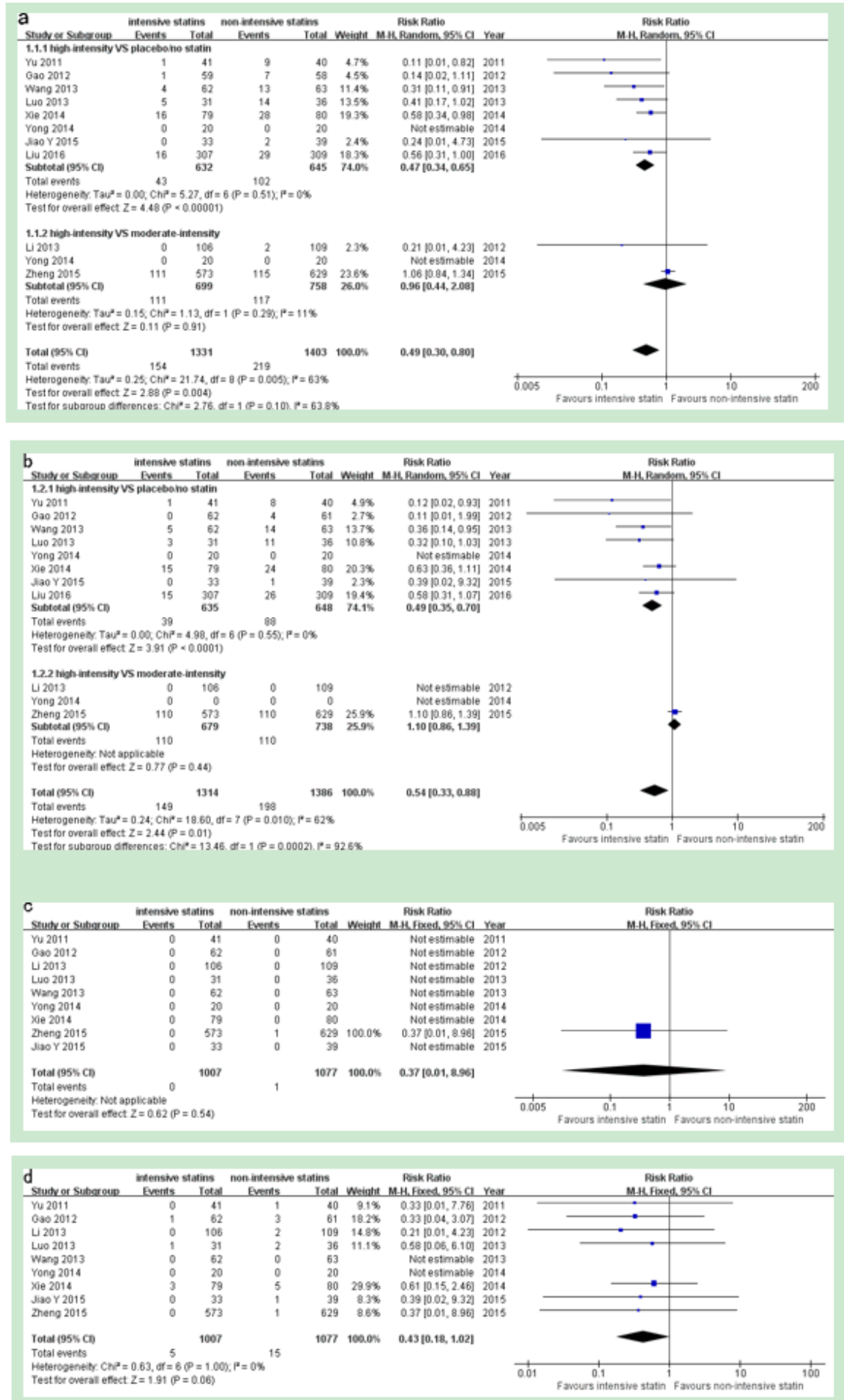

\section{Figure 2}

Forest plot of MACE (a), nonfatal MI (b), cardiac death (c), and TVR (d) with preoperative intensive statin therapy and non-intensive statin therapy in ACS patients. 


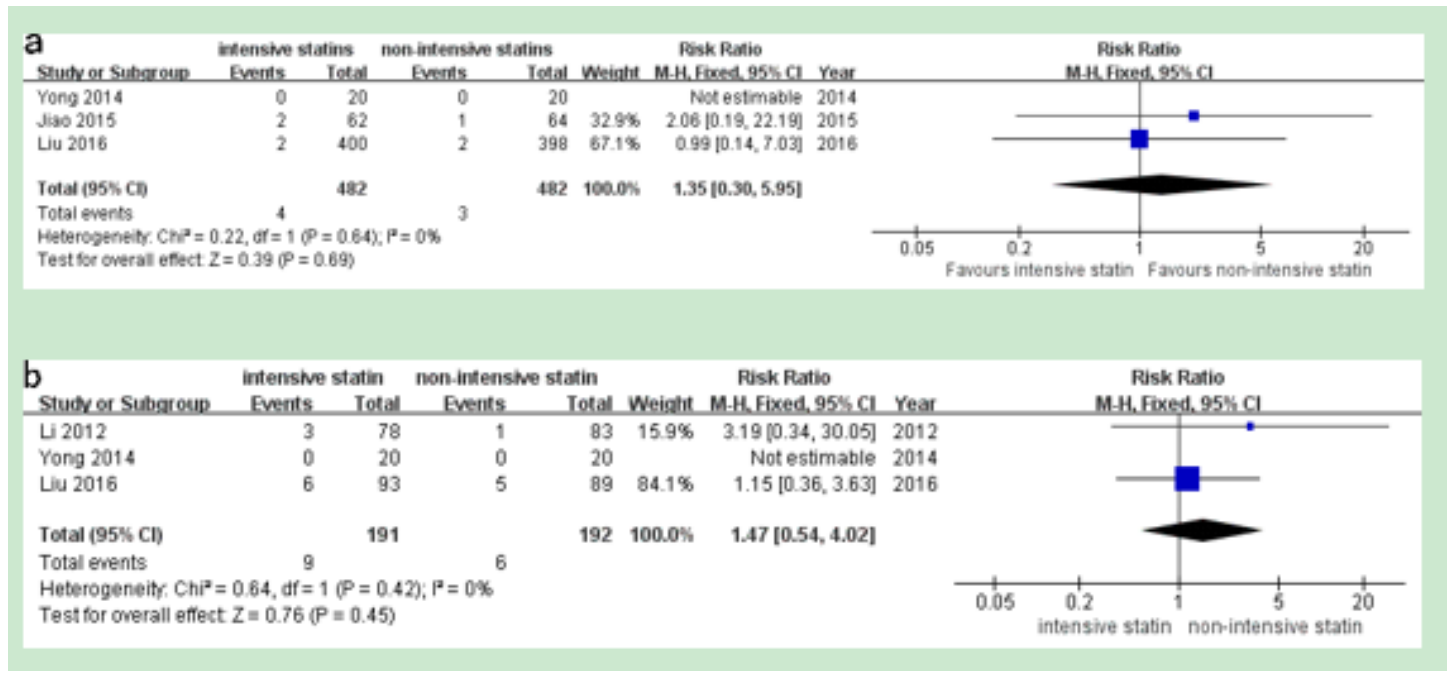

\section{Figure 3}

Forest plot of myalgia/myasthenia (a), and abnormal ALT (b) with preoperative intensive statin therapy and non-intensive statin therapy in ACS patients.

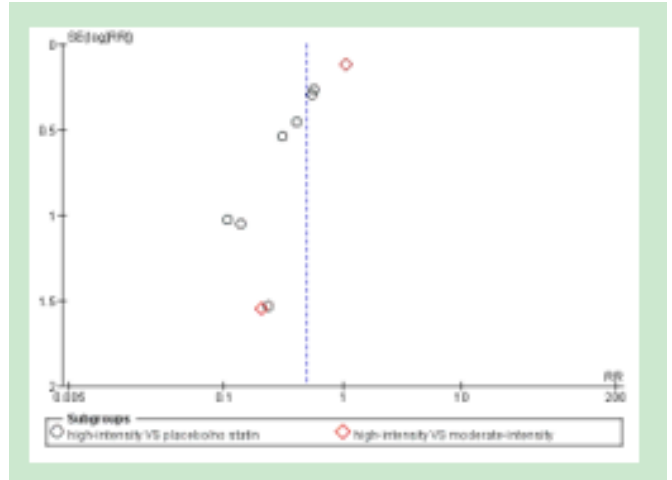

\section{Figure 4}

Funnel plot of MACE with preoperative intensive statin therapy and non-intensive statin therapy in ACS patients

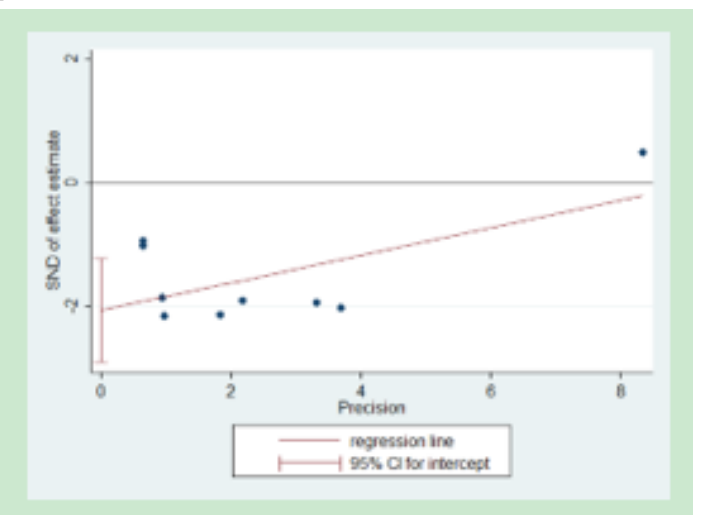

\section{Figure 5}

Egger plot of MACE with preoperative intensive statin therapy and non-intensive statin therapy in ACS patients 


\section{Supplementary Files}

This is a list of supplementary files associated with this preprint. Click to download.

- Additionalfile1.docx 